\title{
Responsabilidad ética de los médicos en Uruguay. Resultados del primer sexenio de funcionamiento del Tribunal de Ética Médica
}

Sylvia Gamero* ${ }^{\star}$ Frances Borches Duhalde ${ }^{\dagger}$, Hugo Rodríguez Almada ${ }^{\ddagger}$

\section{Resumen}

Desde 2012 funciona el Tribunal de Ética Médica creado por la Ley № 18.591, con competencia en todos los casos de ética, deontología y diceología médicas que le sean requeridos por el Estado, personas físicas o jurídicas o por integrantes del Colegio Médico del Uruguay. Este trabajo tiene el propósito de dar a conocer la primera experiencia en Uruguay de juzgamiento de la responsabilidad ética de los médicos regulada por ley.

Objetivos. General: presentar el primer reporte sobre responsabilidad ética de los médicos en Uruguay. Específicos: a) Describir algunas características de las denuncias, los denunciantes y los denunciados por presuntos apartamientos de la ética médica durante los dos primeros períodos de actuación del tribunal. b) Sistematizar las características y los resultados de los procedimientos desarrollados.

Material y método: se analizaron fuentes de información de acceso público: a) Fallos del Tribunal de Ética Médica y del Tribunal de Alzada; b) Memorias del Tribunal de Ética Médica. Se sistematizaron las principales características de las denuncias, los denunciantes, denunciados, los procedimientos y los fallos.

Resultados: se recibieron 101 denuncias, de las que se acogieron 56 (FR: 0,55). Las faltas éticas dentro del equipo de salud fueron el motivo mayoritario. Las denuncias involucraron a 68 médicos, principalmente varones (FR: 0,62) y edad promedio de 52,8 años. Más de la mitad de las denuncias se originó en situaciones asistenciales concretas, especialmente de urgencia/emergencia. Las especialidades quirúrgicas tuvieron alta representación. La mayoría de los denunciados recurrió a asistencia letrada. El tiempo promedio entre la admisión de la denuncia y el fallo definitivo fue de 9,6 meses. Según los fallos definitivos, en la mayoría de los casos (FR: 0,60 ) no existió o no se probó la falta. Fueron sancionados 27 médicos y cinco de ellos fueron suspendidos temporalmente del registro. La mayoría de las faltas fue por mal relacionamiento con otros colegas, pero las más graves fueron por transgresiones en la relación clínica.

Conclusiones: se mantuvo estable el número de las denuncias admitidas. La mayoría de las denuncias y las sanciones aplicadas derivaron de conflictos dentro del equipo de salud. Todas las sanciones de mayor gravedad derivaron de conflictos con los pacientes o sus familias. El perfil de los médicos denunciados es polimorfo, aunque algo más envejecido y masculinizado que el promedio. La actividad de urgencia/emergencia o quirúrgica supondría una mayor exposición a las denuncias, tal como se observa en la responsabilidad civil y penal.

Palabras clave: Uruguay

Ética médica

Responsabilidad legal
Key words: Uruguay

Ethics, medical

Liability, legal

\footnotetext{
* Profesora Adjunta del Departamento de Medicina Legal y Ciencias Forenses. Facultad de Medicina. Universidad de la República. ‡ Asistente del Departamento de Medicina Legal y Ciencias Forenses. Facultad de Medicina. Universidad de la República. ‡ Profesor Titular del Departamento de Medicina Legal y Ciencias Forenses. Facultad de Medicina. Universidad de la República. Departamento de Medicina Legal y Ciencias Forenses. Facultad de Medicina, Universidad de la República, Uruguay. Conflicto de intereses: las Dras. Sylvia Gamero y Frances Borches no presentan conflictos de interés. El Dr. Hugo Rodríguez integró el Tribunal de Ética Médica en el período 8/11/2012 - 8/11/2018.

Correspondencia: Avda. Gral. Flores 2125. Código Postal 11800, Montevideo, Uruguay.

Correo electrónico: hrodriguez@fmed.edu.uy

Recibido: $11 / 3 / 19$

Aprobado: $15 / 4 / 19$
} 


\section{Introducción}

La Ley $N^{\circ} 18.591$ (Creación del Colegio Médico del Uruguay) de 18 de setiembre de 2009 y su reglamentación por el Decreto 83/010 sentaron el régimen de la responsabilidad ética para todos los médicos en Uruguay $^{(1)}$.

La creación del Colegio y su Tribunal de Ética Médica (en adelante TEM) resultó de un largo proceso iniciado en 1943 que incluyó el debate sobre distintos modelos y proyectos de ley ${ }^{(2-7)}$. Previamente existieron otros organismos de control de la profesión en el ámbito administrativo (Comisión de Salud Pública creada por Ley N ${ }^{\circ}$ 9.202 de 12 de enero de 1934), en las organizaciones gremiales (Consejo Arbitral del Sindicato Médico del Uruguay fundado en 1920 y Tribunal de Ética Médica de la FederaciónMédica del Interior fundada en 1966) y a nivel universitario (Comisión de Ética Médica y Conducta Universitaria de la Facultad de Medicina desde $1985)^{(1,9)}$.

El primer TEM creado por ley en Uruguay fue designado el 31 de octubre de 2012 y tuvo su primera sesión el 8 de noviembre del mismo año, tras lo cual aprobó su Reglamento de Procedimiento, en cumplimiento con lo establecido en el artículo 47 del Decreto 83/010 ${ }^{(7)}$. Cesó tras cumplir su período reglamentario de tres años para que tomara posesión e iniciara sus actividades el segundo TEM, que actuó desde el 8 de diciembre de 2015 hasta el 8 de noviembre de $2018^{(10)}$.

Con posterioridad al inicio de las actividades del TEM fue promulgada la Ley No 19.286 (Aprobación del Código de Ética Médica) de 25 de setiembre de 2014. Se trató del primer Código de Ética Médica con rango legal en Uruguay. Sin perjuicio de ello, las faltas éticas que instituye no conforman una lista taxativa sino una base ética mínima y orientativa, ya que el propio texto legal señala que la "enunciación de principios, normas y deberes hecha por este Código no implica el desconocimiento de otros inherentes a los derechos y libertades fundamentales y a las bases éticas de la medicina" $(\text { artículo } 81)^{(4)}$.

Este trabajo tiene el propósito de dar a conocer la primera experiencia en Uruguay de juzgamiento de la responsabilidad ética de los médicos regulada por ley, a partir de la sistematización de los resultados de la actividad cumplida por el TEM durante los dos trienios que comprendió la actuación de las dos primeras administraciones del Colegio Médico del Uruguay.

\section{Objetivos}

General: realizar el primer reporte sobre la responsabilidad ética de los médicos en Uruguay desde la vigencia de la colegiación legal obligatoria.
Específicos:

a) Describir algunas características de las denuncias, los denunciantes y los médicos denunciados por presuntos apartamientos de la ética médica durante los dos primeros períodos de actuación del TEM del Colegio Médico del Uruguay.

b) Sistematizar las características y los resultados de los procedimientos desarrollados a partir de las denuncias promovidas contra los médicos ante el TEM durante el período de referencia.

\section{Material y método}

Se analizaron las siguientes fuentes de información de acceso público correspondientes al período en estudio:

a) Fallos del Tribunal de Ética y del Tribunal de Alzada disponibles en el sitio web del Colegio Médico del Uruguay ${ }^{(10)}$.

b) Memorias del Tribunal de Ética Médica del Colegio Médico del Uruguay ${ }^{(11,12)}$.

Si bien los fallos son publicados con los nombres de los médicos denunciados y de casi todos los denunciantes (salvo situaciones extraordinarias que requieren proteger la intimidad de las víctimas), dados los objetivos de este trabajo, se realizó un manejo anónimo de las partes del proceso, así como de las instituciones y los usuarios involucrados.

De los cuatro casos que estaban en trámite al momento de procesar los datos no se incluyó ningún dato por tratarse de procedimientos con carácter confidencial hasta que exista un fallo firme (artículo 36 de la Ley $\mathrm{N}^{\circ}$ 18.591).

Para conocer la edad y la especialidad de los médicos denunciados, cuando ello no surge de los fallos, se recurrió a la Secretaría del Tribunal de Ética, para lo cual se requirió la autorización del organismo.

Se analizaron las siguientes variables:

\section{Sobre las denuncias:}

a) Número de denuncias: admitidas (culminadas o en proceso) / no admitidas.

b) Tipo de falta ética alegada: en la relación con colegas, el equipo de salud o la institución asistencial / en la relación con usuarios, pacientes o su familia.

c) Contexto del conflicto: asistencial (urgencia/emergencia o policlínica) / no asistencial. Se consideró asistencial si ocurrió en el curso de asistencia a pacientes.

d) Primaria o reconvención (contradenuncia del médico denunciado). 
e) Denuncia realizada por un tercero o autodenuncia (el médico solicitó al tribunal la consideración de una situación que lo involucró).

\section{Sobre los denunciantes:}

a) Personas físicas (médicos/no médicos); personas jurídicas (públicas/privadas).

b) Departamento donde se desarrolló el conflicto.

c) Asistencia letrada: sí/no. Se asumió que las denuncias realizadas por personas jurídicas contaron con asistencia letrada.

\section{Sobre los denunciados:}

a) Edad (medida en años al momento de la denuncia).

b) Sexo (hombre/mujer).

c) Especialidad médica (se consideró la ejercida en el hecho que dio motivo a la denuncia; los denunciados por actos cumplidos en cargos gerenciales se consideraron en la especialidad Administración). d)Asistencia letrada: Sí/No.

\section{Sobre el procedimiento y los fallos:}

a) Duración del procedimiento (medida en meses, desde la fecha en que el TEM acogió la denuncia y la fecha en que se alcanzó un fallo firme).

b) Resultado final del procedimiento: sanción/no sanción.

c) Tipo de sanción: advertencia, amonestación, sanción educativa, suspensión temporal del registro (se indica el lapso), según el artículo 28 de la Ley $\mathrm{N}^{\circ}$ 18.591 .

d) Consensualidad de los fallos del TEM: unanimidad/mayoría.

e) Apelación al fallo del TEM: Sí/No; fallo del Tribunal de Alzada: confirmatorio/revocatorio.

f) Consensualidad de los fallos del Tribunal de Alzada: unanimidad/mayoría.

g) Confirmación de los fallos de suspensión temporal del Registro por el Ministerio de Salud: Sí/No.

\section{Resultados}

Entre el 8 de noviembre de 2012 y el 8 de noviembre de 2018 el TEM recibió 101 denuncias, de las que se acogieron 56 (FR: 0,55) (cuatro de ellas seguían en proceso, estando a consideración del TEM de la tercera administración del Colegio Médico del Uruguay). En el resto de las denuncias, el TEM se declaró incompetente o entendió que la denuncia no cumplió los requisitos establecidos en el Reglamento de Procedimiento.

En la figura 1 se muestra la distribución por año de inicio del procedimiento. Las denuncias admitidas en 2013 incluyen las presentadas en 2012 antes de la designación el primer TEM.

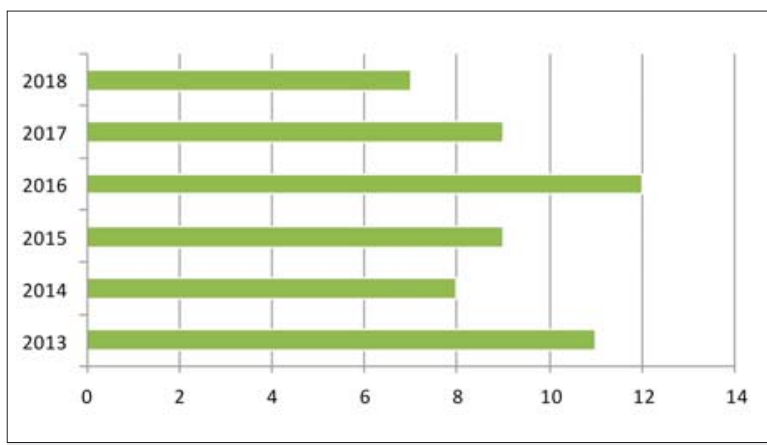

Figura 1. Distribución de los procedimientos por año.

Los motivos alegados en las denuncias admitidas y concluidas $(\mathrm{n}=52)$ fueron faltas éticas en la relación con colegas, equipo de salud o institución ( $n=29, F R: 0,58)$ o en la relación con los usuarios, los pacientes o su familia $(\mathrm{n}=23$, FR: 0,44$)$.

De las 52 denuncias, 31 correspondieron a Montevideo (FR: 0,60). El resto correspondieron a Canelones $(\mathrm{n}=8)$, Rivera $(\mathrm{n}=3)$, San José y Treinta Tres $(\mathrm{n}=2)$ y Colonia, Lavalleja, Maldonado, Paysandú, Salto y Tacuarembó $(\mathrm{n}=1)$.

Hubo 4 denuncias (FR: 0,08) promovidas por los propios médicos involucrados (autodenuncia) y 2 (FR: $0,04)$ reconvenciones (el denunciado contestó el escrito de la denuncia interponiendo una contradenuncia).

Los denunciantes fueron mayoritariamente personas físicas ( $\mathrm{n}=44$, FR: 0,85), principalmente médicos $(\mathrm{n}=33$; FR: 0,75); los 11 restantes fueron pacientes, familiares de pacientes o vecinos del médico denunciado. Hubo ocho denuncias realizadas por instituciones públicas $(n=4)$ o privadas $(n=4)$.

La mayoría de los denunciantes no se presentó con asistencia letrada ( $\mathrm{n}=33$; FR: 0,63). En 19 casos (FR: $0,37)$ los denunciantes recurrieron a asistencia letrada (incluidas las ocho denuncias promovidas por personas jurídicas). Si se consideran solamente las denuncias promovidas por personas físicas, tres de cada cuatro denuncias fueron promovidas sin asistencia letrada.

Las 52 denuncias ya concluidas involucraron a un total de 68 médicos, de los cuales 42 fueron varones (FR: 0,62$)$ y 26 mujeres (FR: 0,38 ). Al momento de la denuncia, los médicos denunciados tenían una edad promedio de 52,8 años (mediana: 55,1; rango: 25,7-72,1 años).

Las especialidades de los médicos denunciados en relación con los hechos alegados se presentan en la tabla 1.

Más de la mitad de las denuncias contra los 68 médi$\cos (n=38$; FR: 0,56$)$ se originó en el curso de situaciones asistenciales concretas. Dentro de ellas se observó 
un franco predominio de las de urgencia/emergencia $(\mathrm{n}=24$; FR: 0,63$)$.

La mayoría de los médicos denunciados recurrió a asistencia letrada durante el proceso ( $n=41$; FR: 0,60). La proporción se mantiene si se cuentan solo los médicos que resultaron sancionados $(\mathrm{n}=27)$ : 16 comparecieron con asistencia letrada (FR: 0,59).

El tiempo promedio transcurrido entre la admisión de la denuncia y el fallo definitivo fue de 9,6 meses (mediana: 9,57; rango: 1,4-20,1 meses), sin descontar el mes de receso anual reglamentario de 30 días del TEM.

Los fallos sobre la conducta de los 68 médicos denunciados fueron apelados en 21 casos (FR: 0,31). Si se consideran solamente los fallos sancionatorios $(\mathrm{n}=31)$, las apelaciones más frecuentes 20 (FR: 0,65). La mayoría de los fallos del Tribunal de Alzada ( $\mathrm{n}=17$; FR: 0,81) ratificaron el de primera instancia. De los cuatro fallos revocatorios del Tribunal de Alzada, tres resultaron más severos que el de primera instancia.

Los fallos de primera instancia sobre los 68 médicos denunciados fueron unánimes en su casi totalidad ( $\mathrm{n}=66$; FR: 0,97). También los fallos de segunda instancia que involucraron a 17 médicos fueron casi todos unánimes (n=16; FR: 0,94).

Según fallos definitivos sobre la conducta de los 68 médicos denunciados, en la mayoría $(n=41 ;$ FR: 0,60$)$ no existió o no se probó la falta ética denunciada.

Las sanciones recibidas por los 27 médicos en que se consideró probada la comisión de las faltas éticas denunciadas se detallan en la tabla 2.

Todos los fallos de los 27 médicos sancionados se fundaron en la Ley No 18.591 y el Decreto 83/010. En 17 de ellos se especificaron transgresiones específicas al Código de Ética Médica de la Ley No 19.286 (se detallan en la tabla 3). Los fallos también tomaron como referencia la Ley $\mathrm{N}^{\circ} 18.335$ (Derechos y obligaciones de pacientes y usuarios de los servicios de salud) y su reglamentación (Decreto 274/010), el Decreto 258/992 (Reglas de conducta médica y derechos de los pacientes), el Código Internacional de Ética Médica de la Asociación Médica Mundial y normas de asociaciones profesionales extranjeras (asociaciones psiquiátricas de Estados Unidos y Canadá).

En dos casos el fallo del Tribunal de Alzada fundó la aplicación de sanciones en la inconducta procesal de los médicos denunciados (escritos de apelación que consideró agraviantes hacia la contraparte o hacia el TEM).

Los fallos firmes de inhabilitación ( $\mathrm{n}=4$ ) fueron elevados al Ministerio de Salud, según lo establecido en la normativa legal vigente, sin que se encontraran apartamientos de la legalidad en el procedimiento cumplido, por lo que quedaron firmes en su totalidad.
Tabla 1. Especialidades médicas (n).

\begin{tabular}{|c|c|}
\hline Administración & 3 \\
\hline Anatomía patológica & 1 \\
\hline Anestesia & 1 \\
\hline Cirugía & 7 \\
\hline Deportología & 1 \\
\hline Emergencia & 8 \\
\hline General & 7 \\
\hline Ginecología & 5 \\
\hline Hemoterapia & 1 \\
\hline $\begin{array}{l}\text { Medicina interna y especialidades médicas (neurología, } \\
\text { endocrinología) }\end{array}$ & 4 \\
\hline Oncología/Radioterapia & 3 \\
\hline Otorrinolaringología & 7 \\
\hline Pediatría & 8 \\
\hline Psiquiatría & 4 \\
\hline Sexología & 1 \\
\hline Trasplantes & 5 \\
\hline Traumatología & 2 \\
\hline
\end{tabular}

\section{Discusión}

Los resultados de los seis primeros años de vigencia del régimen legal de responsabilidad ética de los médicos en Uruguay muestran una sensible estabilidad en cuanto al número de denuncias recibidas.

El alto porcentaje de denuncias no admitidas sugiere un bajo nivel de conocimiento de las competencias legales del TEM y de las formalidades requeridas para su admisibilidad. En efecto, si bien el TEM es competente "para entender en todos los casos de ética, deontología y diceología médicas que le sean requeridos por el Estado, personas físicas o jurídicas o por integrantes del Colegio Médico del Uruguay" (artículo 24 de la Ley N ${ }^{\circ}$ 18.591), no tiene funciones de auditoría, periciales, de recepción de quejas o de consultas ${ }^{(4)}$. Además, en los temas de su competencia, el Reglamento de Procedimiento exige en su artículo 11 que la "denuncia debe contener: a) una exposición clara y detallada de los hechos que se solicitan sean analizados por el Tribunal; b) la individualización del o de los denunciados, cuando ello sea posible; c) la prueba que el denunciante solicita sea incorporada al procedimiento o producida por el Tribunal; d) el objeto de cada prueba y en el caso de prueba 
Tabla 2. Tipo y número de sanciones firmes a los médicos denunciados.

\begin{tabular}{lc}
\hline Tipo de sanción & $n(F R)$ \\
\hline Advertencia & $19(0,70)$ \\
Amonestación & $3(0,11)$ \\
Suspensión del registro por 30 días & $1(0,04)$ \\
Suspensión del registro por 90 días & $2(0,07)$ \\
Suspensión del registro por 6 meses & $1(0,04)$ \\
Suspensión del registro por 6 años & $1(0,04)$ \\
\hline
\end{tabular}

testimonial los hechos sobre los que será interrogado cada testigo; y d) la expresión clara y precisa de lo que se solicita al Tribunal. No se admitirán denuncias anónimas; deberán ser firmadas y contener los datos de identificación, correo electrónico y teléfono del o de los denunciantes". Y que: "El denunciante deberá comparecer personalmente ante el Tribunal a ratificar su denuncia. La inasistencia injustificada se podrá entender como desistimiento de la denuncia presentada". Es factible que la falta de asistencia letrada en la mayoría de las denuncias pueda favorecer su no admisibilidad.

La comprobación de que la mayor parte de las denuncias corresponda a conflictos dentro del propio equipo de salud está en consonancia con la elevada prevalencia de comportamientos disruptivos que se ha reporta$\mathrm{do}^{(13)}$. Que estos conflictos se canalicen en el TEM podría indicar que el recurso de la conciliación está subutilizado. El artículo 10 de la Ley $\mathrm{N}^{\circ} 18.591$ asigna a los Consejos Regionales la función de actuar "como Tribunal de Conciliación frente a los conflictos generados entre miembros del Colegio o de estos con terceros". Podría entenderse la resistencia de los terceros a recurrir a los Consejos Regionales del Colegio Médico para interceder en un conflicto con un médico. De hecho, en las experiencias más exitosas en la aplicación de los métodos alternativos en la resolución de los conflictos entre usuarios y profesionales de la salud no tiene su sede en las organizaciones médicas ${ }^{(14-16)}$. Aun tomando esto en consideración, esta objeción no es de recibo cuando se trata de conflictos entre colegas, que constituyen la mayoría de las denuncias admitidas.

Siendo los conflictos entre los propios médicos o con otro personal de la salud la principal causa de las denuncias, no es de extrañar que el artículo 71 del Código de Ética, que hace referencia al valor de la buena relación entre colegas, haya sido el más empleado como fundamento de las sanciones impuestas. El repaso de las normas mencionadas en los fallos de TEM recuerda que no solo es de aplicación el Código de Ética Médica, sino también otras normas jurídicas, como la Ley $\mathrm{N}^{\mathrm{o}} 18.335$ y su reglamentación (Decreto 274/010), el Decreto 258/992 y, en general, toda la legislacion sanitaria nacional, así como los documentos deontológicos internacionales.

El dato de que la enorme mayoría de las personas físicas denunciantes haya comparecido sin asistencia letrada parece alejar la hipótesis de una intención subyacente de obtener pruebas para una acción judicial. Además, muchos de los procedimientos que culminaron en sanciones se basaron en hechos previamente judicializados. En este sentido, parece importante reconocer que los conflictos de los médicos con sus colegas, las instituciones asistenciales, los usuarios y sus familias, y los reclamos que desencadenan, tienen una base real multifactorial sobre la que se puede actuar en beneficio de un mejor ejercicio profesional ${ }^{(1,13,14,19)}$.

La alta participación de Montevideo en el total de las denuncias está en sintonía con la desigual distrubuición de los médicos en el país ${ }^{(20)}$. El universo estudiado no permite aún un análisis más afinado por región o por departamento.

El perfil etario de los denunciados (media: 52,8 años; mediana: 55,1 años) es algo más envejecido que el promedio de los médicos en ejercicio ${ }^{(20)}$. En coincidencia con lo que desde hace 20 años ya mostraba la experiencia de juzgamiento ético en Brasil, esa población se compone mayoritariamente de médicos en el pleno ejercicio de la profesión ${ }^{(21)}$. De modo que, paradójicamente, tener experiencia de años puede ser un factor de riesgo, tanto de responsabilidad ética como jurídica. Este fenómeno podría estar relacionado con condiciones de ejercicio profesional que perjudiquen la calidad de relación clínica y favorezca la generación de episodios disrupti$\operatorname{vos}^{(13,20)}$.

Además, llama la atención que casi dos tercios de los denunciados sean varones, en contraste con el perfil de la población médica en Uruguay, crecientemente feminizada, que en el sexenio en estudio muestra entre un $60 \%$ y $70 \%$ de mujeres. ${ }^{(20,22,23)}$.

El hecho de que el envejecimiento de la población médica varía en sentido inverso que su feminización podría explicar, al menos en parte, el predominio de los varones entre los denunciados.

También se debe considerar que la distribución de la población médica por especialidad no es igual para los varones y las mujeres. En cuanto a las especialidades más involucradas en los hechos denunciados, este primer reporte muestra una sobrerrepresentación de las especialidades quirúrgicas en relación con su participación en el total de los profesionales y de los actos médi$\cos ^{(22,23)}$. Pero, sobre todo muestra que las actividades de urgencia/emergencia, sean prehospitalarias u hospitala- 
Tabla 3. Artículos del Código de Ética Médica mencionados en los fallos sancionatorios y número de menciones

Artículo Texto $\quad$ No

$2^{\circ} \quad$ a) Los profesionales de la medicina deben cuidar la salud de las personas y de la comunidad sin discriminación de clase alguna, respetando

menciones

integralmente los derechos humanos.

b) Es deber fundamental prevenir la enfermedad y proteger y promover la salud de la colectividad.

c) El médico debe ejercer inspirado por sentimientos humanitarios. Jamás actuará para generar torturas, tratos crueles, inhumanos 0 degradantes, ni para el exterminio del ser humano, ni para cooperar o encubrir atentados contra la integridad física o moral de sus semejantes. d) El médico, en el marco de su actuación profesional debe promover las acciones necesarias para que el ser humano se desarrolle en un ambiente individual y socialmente sano. Para ello se basará en una formación profesional reconocida y se guiará por las normas y principios éticos establecidos en este Código.

e) El médico debe procurar siempre el más alto nivel de excelencia de conducta profesional.

$3^{\circ} \quad$ Es deber del médico, como profesional de la salud, seguir los siguientes principios y valores fundamentales:

a) Respetar la vida, la dignidad, la autonomía y la libertad de cada ser humano y procurar como fin el beneficio de su salud física, psíquica y social.

b) No utilizar el ejercicio profesional para manipular a las personas desde un punto de vista de los valores.

c) Posibilitar al paciente el encuentro con otro profesional idóneo si él no está en condiciones de ayudarle dentro de sus conocimientos específicos.

d) Hacer, como profesional de la salud y como miembro del Colegio Médico del Uruguay, todo lo que esté dentro de sus posibilidades para que las condiciones de atención sanitaria sean las más beneficiosas y no estigmatizantes para sus pacientes y para la salud del conjunto social sin discriminación alguna.

e) Respetar el derecho del paciente a guardar el secreto sobre aquellos datos que le pertenecen y ser un fiel custodio, junto con el equipo de salud, de todas las confidencias que se le brindan, las que no podrá revelar sin autorización expresa del paciente.

f) Mantenerse al día en los conocimientos que aseguren el mejor grado de competencia profesional en su servicio específico a la sociedad. g) La búsqueda de lucro económico u otros beneficios nunca deberá ser la motivación determinante en su forma de ejercer la profesión. Asimismo, no deberá permitir que motivos de orden económico u otros intereses influyan en la recomendación profesional referida a sus pacientes, procurando también que la provisión de medios idóneos de diagnóstico y tratamiento sean éticamente adecuados.

h) Ser veraz en todos los momentos de su labor profesional, para que los pacientes y la sociedad tomen las decisiones que les competen. i) Concertar y utilizar el progreso científico y tecnológico de la medicina de tal manera que el humanismo esencial de la profesión no resulte desvirtuado.

j) Valorar el trabajo de equipo tanto en su labor de servicio a la salud de sus pacientes como de la población en general.

$4^{\circ} \quad$ El médico tiene responsabilidad en la calidad de la asistencia tanto a nivel personal, como en promoverla a nivel institucional. Es su deber exigir las condiciones básicas para que ella sea garantizada efectivamente en beneficio de las personas, así como reclamar ante los organismos competentes si persisten las condiciones insuficientes en las instituciones. Los médicos que ocupen cargos de dirección deberán proporcionar a los médicos prestadores de la asistencia los recursos humanos y de infraestructura necesarios para que el servicio se preste adecuadamente.

$15^{\circ} \quad$ La historia clínica es un documento fundamental en el acto médico, de ahí que:

a) El médico tiene el deber y el derecho de registrar el acto médico en una historia clínica, que pertenece al paciente pero que quedará bajo la custodia del médico tratante o de la institución de la que es usuario.

b) El paciente tiene derecho al acceso a su historia y a obtener del médico un informe completo y veraz sobre su enfermedad y la asistencia que se le ha brindado.

$18^{\circ}$ Se considera falta ética toda publicidad engañosa o desleal. El médico no debe inducir a engaño a la sociedad propiciando procedimientos 0 productos comerciales cuya eficacia no está comprobada científicamente.

24 El ejercicio clínico de la medicina requiere el vínculo directo con el paciente. La complementación de la asistencia médica a distancia a través de los medios de comunicación, como la telemedicina, seguirá los principios de este Código.

$26^{\circ} \quad$ Todo médico tiene el deber de:

a) Guardar y respetar la intimidad del cuerpo y de las emociones del paciente cuando es interrogado, examinado o tratado.

$29^{\circ} \quad$ El médico deberá siempre respetar al ser humano que ha confiado en él. Los actos médicos que emprenda no serán nunca simples gestos técnicos, sino que se integrarán con todos los valores esenciales de la relación médico-paciente.

$69^{\circ} \quad$ a) Como investigador, el médico nunca suprimirá datos discordantes con sus hipótesis o teorías, ni falsificará ni inventará datos. La debida transparencia y obligada declaración pública ante potenciales conflictos de intereses no solo representa una salvaguarda de la relación entre médico y paciente, sino que también de la confianza que el público en general deposita en la profesión médica y en la investigación biomédica. b) En su comunicación científica no ocultará los aportes recibidos de otros autores ni intentará minimizar los méritos de estos. Nunca se atribuirá trabajos que no hayan sido realizados por él. El plagio científico es una falta ética.

c) El médico debe comunicar sus hallazgos científicos en un ambiente calificado para valorarlo. Solo después podrá divulgarlo públicamente. Será objetivo y veraz, no creando falsas esperanzas ni sobrevalorando sus hallazgos.

$70^{\circ} \quad$ Los datos obtenidos en investigaciones son confidenciales y solo se puede revelar la identidad del sujeto de investigación con autorización expresa de este. Las comunicaciones y publicaciones deben garantizar el anonimato de los integrantes de la población investigada.

$71^{\circ}$ La buena relación humana entre los colegas es fundamental por su valor en sí misma, por su repercusión en la asistencia de los pacientes y para la convivencia en el ámbito de trabajo colectivo. No son éticas la difamación y la injuria ni los comentarios capaces de perjudicar al colega en el ejercicio de su profesión, más allá de las consideraciones que pueda hacer la Justicia.

810 La enunciación de principios, normas y deberes hecha por este Código no implica el desconocimiento de otros inherentes a los derechos y libertades fundamentales y a las bases éticas de la medicina. 
rias, son las más expuestas a las denuncias. Ambas actividades se consideran de mayor riesgo en materia de reclamos por responsabilidad médica civil y penal.

Que la tercera parte de los médicos que resultaron sancionados (y la mitad de quienes recibieron las sanciones más graves con suspensión temporal del registro) optara por comparecer sin abogado durante el proceso podría estar indicando un error de percepción sobre la importancia de la denuncia o una suerte de negación de la situación denunciada. En igual sentido, es significativo que dos de los cuatro médicos que se presentaron espontáneamente ante el TEM para que se valorara su conducta recibieron una sanción.

El tiempo promedio de los procedimientos ha sido de 9,6 meses, lo que supone una duración bastante extensa pero dentro de los plazos procesales. En consonancia con el principio del derecho a un proceso de duración razonable, el artículo 45 del Decreto 83/010 establece que la instrucción deberá quedar finalizada dentro de 180 días hábiles. Se debe tomar en cuenta que a la duración promedio de 9,6 meses se le debe descontar un mes completo por el período de receso reglamentario del TEM durante el cual se interrumpen los plazos, de tal modo que el tiempo procesal promedio real fue de 8,6 meses. Además, el mismo artículo 45 establece que el plazo es prorrogable por 60 días en función de la complejidad del caso y de la obtención de la prueba, muchas veces dependiente de testigos que no tienen obligación de comparecer y de los tiempos demandados por las pruebas por oficio solicitadas a organismos públicos e instituciones privadas. Por último, el Reglamento de Procedimiento, de corte muy garantista, otorga a las partes la posibilidad de solicitar prueba complementaria por 30 días, derecho a alegar (10 días), vista previa en todos los casos en que recaiga alguna sanción y derecho al recurso de alzada ( 10 días para interponerlo y 30 para producir el fallo definitivo), según lo establecido en el artículo 30 de la propia Ley $\mathrm{N}^{\circ} 18.591$.

Resulta significativo que la mayoría de los fallos del TEM no motivaron un recurso de revocación ante el Tribunal de Alzada. Como era esperable, entre los médicos sancionados en primera instancia las apelaciones fueron más frecuentes. Además, no solo la mayoría de los fallos de alzada ratificaron los emitidos por el TEM, sino que la casi totalidad fueron suscritos por unanimidad de sus cinco miembros, tanto en primera como en segunda instancia.

Las posibles sanciones están limitadas a lo establecido en la norma legal: a) advertencia, b) amonestación, c) sanción educativa y d) suspensión temporal en el registro por un máximo de diez años. El tenor de la gran mayoría de las sanciones aplicadas (advertencia) parece indicar una intención educativa hacia el colectivo médico, con una penalización de carácter moral. Solamente en cinco casos el TEM entendió que la gravedad de la transgresión de los médicos justificaba la suspensión temporal, uno de los cuales fue revocado por el Tribunal de Alzada. Todos los casos que culminaron con sanción grave de suspensión temporal se originaron en denuncias por conflictos de los médicos con los pacientes o sus familiares. Más allá de lo cuestionable de la denominación legal de la "sanción educativa", es posible que su implementación efectiva requiriera alguna forma de reglamentación.

\section{Conclusiones}

El análisis de los primeros seis años de vigencia del régimen legal de responsabilidad ética de los médicos en Uruguay muestra una estabilidad en el número de las denuncias admitidas.

La mayoría de las denuncias y las sanciones aplicadas derivaron de conflictos dentro del equipo de salud. No obstante, las sanciones más graves, que suponen la suspensión temporal del registro, derivaron de conflictos con los pacientes o sus familias.

El universo de los médicos denunciados es polimorfo, aunque algo más masculinizado y envejecido que el promedio de la profesión, con experiencia de varios años de ejercicio y mayor exposición para los que realizan actividades de urgencia/emergencia o quirúrgica.

\section{Abstract}

The Medical Ethics Tribunal has been functioning since 2012 when it was created by Act 18,591 for all cases in connection with medical ethics, deontology and diceology required by the government, individuals or legal persons, or members of the Uruguayan Medical Association. The study aims to inform about the first experience in Uruguay in the judging of the ethical responsibility of medical doctors ruled by law.

Objective: General: To present the first report on ethical responsibility of medical doctors in Uruguay.

Specific: a) To describe a few characteristics of the complaints, the people who report the cases and those who are accused for alleged violation of medical ethics in the first two terms of the Tribunal's functioning. $b$ ) to systematize the characteristics and results of the procedures developed.

Method: the public access sources of information were analysed: a) judgements by the Ethics Tribunal and the Appeals Court; b) Memories of the Medical Ethics Tribunal. A few characteristics of the reports were systematized, as well as those in connection with the people who report the cases and those who are accused for alleged violation, the procedures and the judgements. 
Results: 101 complaints were received, 56 of which were accepted (FR: 0.55). Ethical offences by members of the health team were the most frequent reason. Complaints involved 68 doctors, mainly male (FR: 0.62 ) average age being 52.8 years old. Over half the complaints originated in specific situations where medical services were being provided, in particular in the ER context. Surgical procedures were highly represented. Most accused doctors appeared before the tribunal. Average time between the acceptance of the complaint and the final judgement was 9.6 months. According to the final judgments, in most cases (FR: 0.6) there was no offence or the offence could not be proved. 27 doctors were punished and 4 of them were temporarily excluded from the registry. Most offences involved a bad relationship with colleagues, although the most serious ones consisted in abusing the clinical relationship.

Conclusions: the number of complaints received was stable. Most complaints and the punishments applied originated in problems within the health team. All greater punishments originated in conflicts with patients or their families. The profile of doctors reported is somehow more aged and male individuals than the polymorph average. Activity in the ER and/or the OR would imply being more exposed to complaints, just like it is seen in civil and criminal liability cases.

\section{Resumo}

O Tribunal de Ética Médica criado pela Lei No 18.591 funciona desde 2012 com competência em todos os casos de ética, deontologia e diceologia médicas que sejam requeridos pelo Estado, por pessoas físicas ou jurídica ou por integrantes do Colégio Médico del Uruguay. O objetivo deste trabalho é apresentar a primeira experiência no Uruguai de julgamento da responsabilidade ética dos médicos regulada por lei.

Objetivos: Geral: apresentar o primeiro relatório sobre responsabilidade ética dos médicos no Uruguai. Específicos: a) Descrever algumas características das denúncias, dos denunciantes e dos denunciados por supostos afastamentos da ética médica durante os dois primeiros períodos de trabalho do tribunal. b) Sistematizar as características e os resultados dos procedimentos realizados.

Material e método: foram analisadas fontes de informação de acesso público: a) Sentenças do Tribunal de Ética e do Tribunal de Alçada; b) Memórias do Tribunal de Ética Médica. As principais características das denúncias, dos denunciantes, denunciados, dos procedimentos e das sentenças foram sistematizadas.

Resultados: foram recebidas 101 denúncias, das quais 56 (FR: 0,55) foram aceitas. Na maioria delas os motivos foram faltas éticas dentro da equipe de saúde.
As denúncias incluíam 68 médicos, principalmente do sexo masculino (FR: 0,62) com idade média 52,8 anos. Mais da metade das denúncias foram originadas em situações assistenciais concretas, especialmente de urgência/emergência. As especialidades cirúrgicas tiveram uma alta incidência de casos. A maioria dos denunciados solicitou assistência letrada. A média do período entre a admissão da denúncia e a sentença definitiva foi de 9,6 meses. De acordo com as sentenças definitivas, na maioria dos casos (FR: 0,60) a falta não existiu ou não foi provada. Foram sancionados 27 médicos e 4 foram suspendidos temporariamente do registro. A maioria das faltas foi por mal relacionamento com outros colegas, porém as mais graves foram por transgressões na relação clínica.

Conclusões: o número de denúncias admitidas foi estável durante o período estudado. A maioria delas e das sanções aplicadas estavam relacionadas com conflitos dentro de equipe de saúde. Todas as sanções de maior gravidade foram originadas por conflitos com os pacientes ou com suas famílias. O perfil dos médicos denunciados é um pouco mais envelhecido e masculinizado do que a média polimorfa. A atividade de urgência/emergência e/ou cirúrgica supõem uma maior exposição às denúncias, como se observa na responsabilidade civil e penal.

\section{Bibliografía}

1. Rodríguez Almada H. Introducción a la responsabilidad profesional de los médicos y las instituciones asistenciales. En: Medicina Legal. Derecho Médico. Montevideo: Oficina del Libro-FEFMUR, 2017:101-10.

2. Turnes A. Desarrollo de la Colegiación Médica en Uruguay. Noticias 2009; (153):32-3. Disponible en: https://www. smu.org.uy/publicaciones/noticias/noticias153/art18.pdf [Consulta: 1 de febrero 2019].

3. França O. Hacia el primer código uruguayo de ética médica. Rev Méd Urug 1989; (5):3-10.

4. Rodríguez Almada H. Ley $\mathrm{N}^{\circ} 19.286$ : un hito para la profesión médica y la sociedad uruguaya. Rev Méd Urug 2014; 30(4):217.

5. Torres Calvete J. Colegio Médico del Uruguay. Rev Méd Urug 2012; 28(2):87-8.

6. Giuiria F. Roles de la Colegiación. En: Jornada de Prevención de la Malpraxis Médica, I (1992). Montevideo: SMU, 1993:59-61.

7. Carámbula M. Perspectivas parlamentarias del proyecto de Colegiación. En: Jornada de Prevención de la Malpraxis Médica, I (junio). Montevideo: SMU, 1993:62-4.

8. Lombardi R. Colegiación médica. Noticias 2010; (156):29-30. Disponible en: https://www.smu.org.uy/publicaciones/noticias/noticias156/art9.pdf [Consulta: 2 de marzo 2019]. 
9. Adriasola G. La autorregulación corporativa en el Uruguay. En: Responsabilidad médica y ley penal. Montevideo: Del Foro, 1998:35-7.

10. Colegio Médico del Uruguay. Fallos emitidos por el Tribunal de Ética. Disponible en: https://www.colegiomedico.org.uy/fallos-emitidos-por-el-tribunal-de-etica/ [Consulta: 1 de febrero 2019].

11. Colegio Médico del Uruguay. Memoria ejercicio 2012-2015. Disponible en: https://www.colegiomedico.org.uy/wp-content/uploads/2018/07/ejercicio-2012-2015-1-1.pdf [Consulta: 1 de febrero 2019].

12. Colegio Médico del Uruguay. Memoria del CMU 2015-2018. Montevideo: CMU, 2018. Disponible en: https://www.colegiomedico.org.uy/wp-content/uploads/2018/08/Memoria-del-CMU-2015-2018.pdf [Consulta: 1 de febrero 2019].

13. Godino M, Barbato M, Ramos L, Otero M, Briozzo L. Encuesta Nacional de Comportamientos Disruptivos en el Equipo de Salud. Rev Méd Urug 2014; 30(4):235-46.

14. Sindicato Médico del Uruguay, Comisión de Legislación Sanitaria y Derecho Médico. Métodos alternativos para la resolución de conflictos entre usuarios y prestadores de servicios de salud: conciliación y mediación. Montevideo: SMU, 2001: $60 \mathrm{p}$

15. Comisión Nacional de Arbitraje Médico. Memorias del V Simposio Internacional: por la calidad de los servicios médicos y la mejoría de la relación médico-paciente. México D.F.: CONAMED, 2000: 180p.
16. Etxeberría Gabilondo F. Alternativas extrajudiciales en la responsabilidad médica: la experiencia del arbitraje médico en España. En: Rodríguez Almada H, coord. Derecho Médico. Montevideo: Bdef, 2001:305-15.

17. Ramírez A. La experiencia de la Comisión Nacional de Arbitraje Médico de México. En: Rodríguez Almada H, coord. Derecho Médico. Montevideo: Bdef, 2001:331-42.

18. Rodríguez Almada H. Los aspectos críticos de la responsabilidad médica y su prevención. Rev Méd Urug 2001; 17:17-23.

19. Rodríguez Almada H. De la medicina defensiva a la medicina asertiva. Rev Méd Urug 2006; 22(3):167-8.

20. González Mora F, Barbero Portela M, Barrero Salgado G, Batthyany Dighiero K, coord. La profesión médica en Uruguay: caracterización del perfil profesional y la inserción laboral de los médicos en Uruguay. Montevideo: Colegio Médico del Uruguay, 2018:121p.

21. Gomes JC, Drumund JG, França GV. Erro Médico. 3 ed. Montes Claros: Unimontes, 2001:222 p.

22. Sindicato Médico del Uruguay. Demografía médica en el Uruguay. Disponible en: https://www.smu.org.uy/sindicales/documentos/demografia.html [Consulta: 8 de oct 2018].

23. Caja de Jubilaciones y Pensiones de Profesionales Universitarios. Afiliados activos al 31.12.2017, por edad sexo y profesión. Disponible en: http://www.cjppu.org.uy/download.php? $\mathrm{m}=\mathrm{g} \& \mathrm{i}=886$ [Consulta: 8 de marzo 2019]. 